KFA-IKP(TH)-97-12

\title{
The BFKL-Regge Expansion for the Proton Structure Function at Small $x$
}

\author{
Vladimir R. Zoller \\ Institute for Theoretical and Experimental Physics \\ 117218 Moscow, Russia ${ }^{1}$
}

\begin{abstract}
We report an evaluation of subleading eigenvalues and eigenfunctions of the BFKL equation in the color dipole representation with a running gauge coupling. We present an expansion of the small- $x$ proton structure function $F_{2 p}\left(x, Q^{2}\right)$ in terms of the rightmost BFKL-singularities. The BFKL-Regge phenomenology of DIS structure functions is developed which is shown to provide remarkably good description of the data on $F_{2 p}\left(x, Q^{2}\right)$ from E665 to HERA.
\end{abstract}

\section{Introduction}

In this talk I address the issue of extrapolation of the proton structure functions to a domain of very small $x$ from the attainable kinematical region of $x$ and $Q^{2}$. So, this is an old problem of predicting the future from the known past. The standard predictor is the GLDAP evolution equation [1]. However, the art of predicting is difficult. The numerous pre-HERA GLDAP fits to the proton structure functions are equally good for large- $x$-data but seldom come close to the new data points at smaller $x$. The lack of predictive power is not surprising and we comment on this point below. It is well known that the GLDAP evolution breaks down at small $x$ and is superseded by the $\log (1 / x)$-BFKL-evolution [2]. Contrary to the GLDAP approach, the BFKL evolution predicts uniquely the proton structure functions at an arbitrarily small $x$ from the input at a starting point $x=x_{0}$. DIS structure functions at asymptotically large $1 / x$ are dominated by the rightmost Regge-singularity with the intercept $\Delta_{0}[3]$ and

$$
F_{2 p}\left(x, Q^{2}\right)=F_{2}^{(0)}\left(Q^{2}\right)\left(\frac{1}{x}\right)^{\Delta_{0}}
$$

1) Talk at 5th International Workshop on Deep Inelastic Scattering and QCD (DIS'97), Chicago, April 1997 
At moderate $x$, however, the subleading contributions to $F_{2 p}\left(x, Q^{2}\right)$ with smaller intercepts $\Delta_{1}, \Delta_{2}, \ldots$ can not be neglected. The task of my talk is to present an evaluation of the intercepts $\Delta_{n}$, the pomeron trajectory slopes $\alpha_{n}^{\prime}$ and the corresponding structure functions $F_{2}^{n}\left(Q^{2}\right)$, to arrive finally at the representation

$$
F_{2 p}\left(x, Q^{2}\right)=\sum_{n} A_{n} F_{2}^{(n)}\left(Q^{2}\right)\left(\frac{x_{0}}{x}\right)^{\Delta_{n}}
$$

I conclude with the expansion of the proton structure function $F_{2}\left(x, Q^{2}\right)$ in terms of the three rightmost BFKL singularities. Such a three pole approximation seems to exhaust the existing experimental data thus providing a reliable basis for the BFKL-Regge phenomenology of diffractive DIS.

\section{The BFKL Eigenvalue Problem in the Dipole Picture}

The virtual photo-absorption cross section $\sigma(r, x)$, where $r$ is the color dipole size, satisfies the BFKL equation [4] (hereafter $\xi=\log (1 / x)$ ):

$$
\frac{\partial \sigma(\xi, r)}{\partial \xi}=\mathcal{K} \otimes \sigma(r, x)
$$

with the kernel $\mathcal{K}$ which involves the running gauge coupling and the infrared cutoff- the correlation radius $R_{c}$ of perturbative gluons. We look for the solution with the Regge behavior

$$
\sigma_{n}(r, x)=\sigma_{n}(r)\left(\frac{1}{x}\right)^{\Delta_{n}}
$$

Then the eigenfunctions $\sigma_{n}(r)$ and the eigenvalues $\Delta_{n}$ are determined from

$$
\mathcal{K} \otimes \sigma_{n}(r)=\Delta_{n} \sigma_{n}(r) .
$$

The short-distance asymptotics of the eigenfunctions is known in the analytic form $[5]$

$$
\sigma_{n}(r)=r^{2}\left[\frac{1}{\alpha_{S}(r)}\right]^{\gamma_{n}-1},
$$

where $\gamma_{n} \Delta_{n}=4 / 3$. At large distances, $r \gg R_{c}$,

$$
\sigma_{n}(r) \equiv \bar{\sigma}_{n}=\text { const }
$$

due to the finite $R_{c}$. Another useful clue is that the leading eigenfunction $\sigma_{0}(r)$ is node free and the $n$-th subleading solution must have $n$ nodes. Then a practical approach to the eigenvalue problem is a variational procedure [6] applied to a class of $n$-node polynomials $\mathcal{P}_{n}(z)$ in a variable $z \sim\left[1 / \alpha_{S}(r)\right]^{\gamma}$. 


\section{Results and Discussion}

The BFKL equation with running coupling and infrared cutoff has a discrete spectrum. The eigenvalues $\Delta_{n}$ obtained by the variational method for $n=$ $0,1,2,3, \ldots$ are as follows

$$
\Delta_{n}=0.40,0.220,0.148,0.111,0.088,0.073,0.063, \ldots
$$

To an accuracy better than $10 \%$ the above series follows the law

$$
\Delta_{n}=\frac{\Delta_{0}}{(n+1)}
$$

derived by Lipatov [3] from quasi-classical considerations.

The BFKL eigenfunctions are represented (Figure 1) in term of the quantity $\sigma_{n}(r) / r$ which to a crude approximation is similar to Lipatov's quasi-classical eigenfunctions, which are $\mathcal{E}_{n}(r) \sim \cos [\phi(r)]$ for $n \gg 1[3]$.

Once $\sigma_{n}(r)$ is known, the pomeron trajectory slope $\alpha_{n}^{\prime}$ can readily be derived. In the notations of ref. [7]

$$
\alpha_{n}^{\prime}=\frac{3}{32 \pi R_{c}^{2}} \int d \rho^{2} \rho^{2} \alpha_{S}(\rho) K_{1}^{2}\left(\rho / R_{c}\right)\left[1+\sigma_{n}(\rho) / \bar{\sigma}_{n}\right]
$$

where (see eq.7)

$$
\bar{\sigma}_{n}=\frac{3}{2 \pi R_{c}^{2}} \int d \rho^{2} \alpha_{S}(\rho) K_{1}^{2}\left(\rho / R_{c}\right) \sigma_{n}(\rho) .
$$

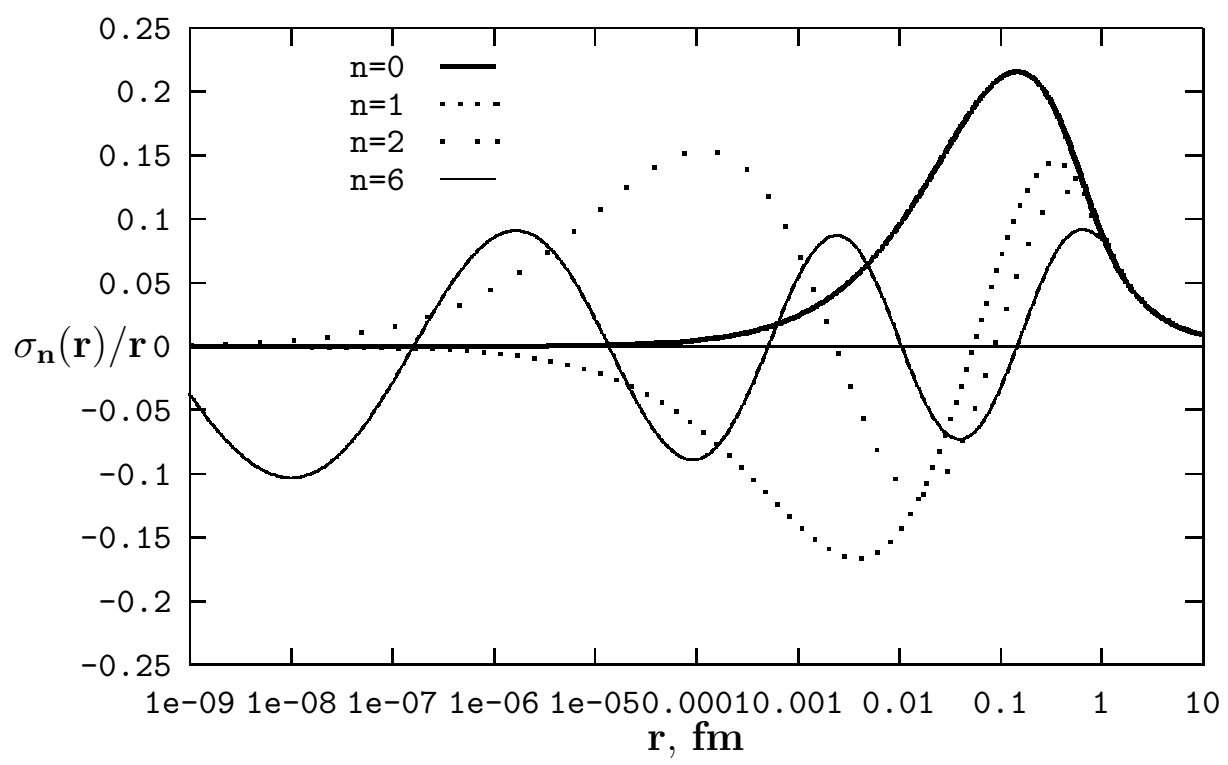

FIGURE 1. BFKL eigenfunctions. 
Numerically, $\alpha_{0}^{\prime}=0.072 \mathrm{GeV}^{-2}, \alpha_{1}^{\prime}=0.066 \mathrm{GeV}^{-2}, \alpha_{2}^{\prime}=0.062 \mathrm{GeV}^{2}, \alpha_{3}^{\prime}=$ $0.060 \mathrm{GeV}^{2}$.

The color dipole factorization relates the dipole cross sections with the structure functions $F_{2}^{(n)}\left(Q^{2}\right)$ which are shown in Figure 2. At large $Q^{2}$, far beyond the nodal region,

$$
F_{2}^{n}\left(x, Q^{2}\right) \propto\left(\frac{x_{0}}{x}\right)^{\Delta_{n}}\left[\frac{1}{\alpha_{S}\left(Q^{2}\right)}\right]^{4 / 3 \Delta_{n}}
$$

Since the relevant variable is a power of the inverse gauge coupling the nodes are spaced by 2-3 orders of magnitude in $Q^{2}$-scale and only the first two of them are in the accessible range of $Q^{2}$. The first nodes of $F_{2}^{(n)}\left(Q^{2}\right)$ are located at $Q^{2} \sim 20-60 \mathrm{GeV}^{2}$. Hence, only the leading structure function contributes significantly in this region. This explains the precocious BFKL asymptotics found from the numerical solution of the BFKL equation [8].

In the accessible range of $x$ the subleading contributions are numerically large. In particular, the BFKL expansion of the Born approximation dipole cross section which is used as the boundary condition at $x=x_{0}=0.03$ suggests more than $60 \%$ contribution from $F_{2}^{(n)}$ with $n>0$. This implies the subleading terms determine the $Q^{2}$ dependence of $F_{2 p}\left(Q^{2}\right)$ at $x=x_{0}$ and simultaneously the $x$ dependence of the structure functions. Notice that in the pre-nodal region of $Q^{2} \lesssim 20 \mathrm{GeV}^{2}$ the leading and subleading structure functions are very similar in shape. This explains the failure of the early GLDAP fits: only the limited region of $Q^{2} \lesssim 10 \mathrm{GeV}^{2}$ was accessible and they

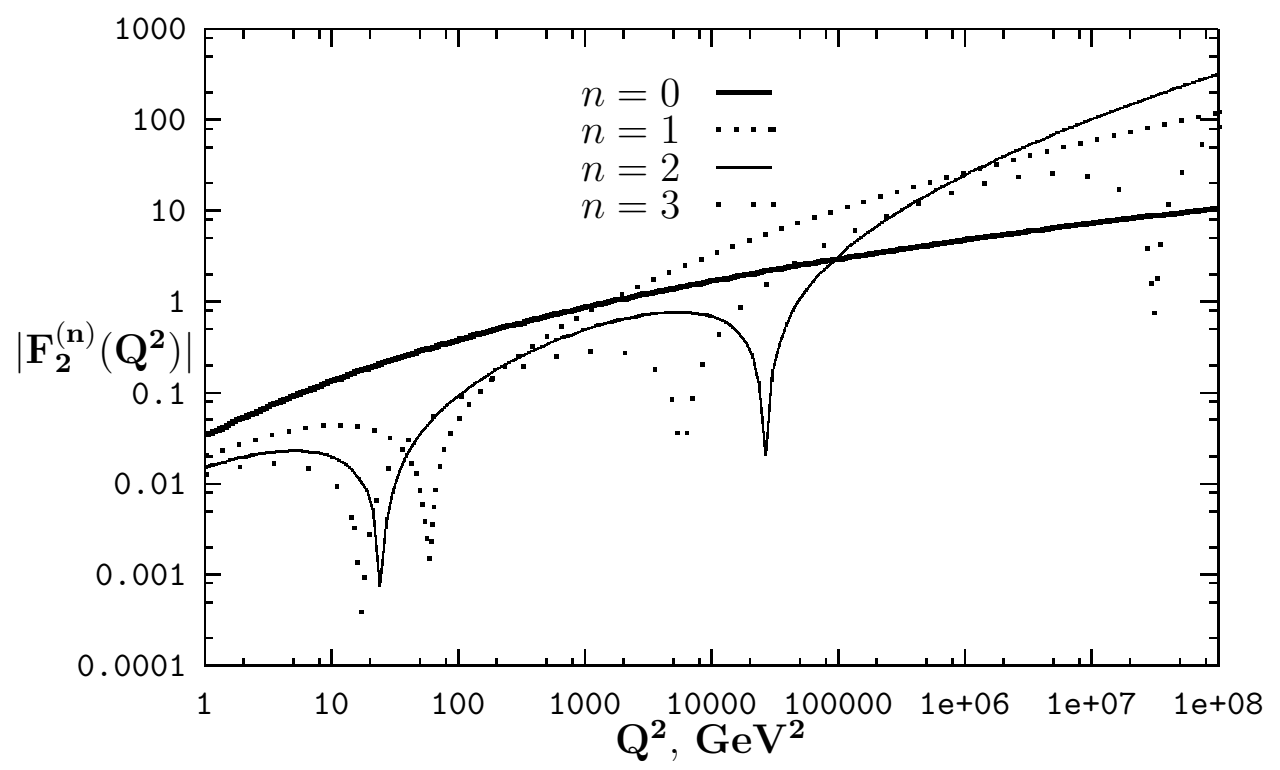

FIGURE 2. The modulus of structure function $F_{2}^{(n)}\left(Q^{2}\right)$ for $n=0,1,2,3$ 
could not catch and correctly describe a very different $x$-evolution of pomerons with different $n$.

At small $x$ only the region $Q^{2} \lesssim 10^{3} \mathrm{GeV}^{2}$ is accessible. In this range the structure functions with $n \geq 3$ are hardly distinguishable. Besides, the splitting of the intercept with $n \geq 3$ is much smaller than for $n=0,1,2$. Hence, the Regge expansion (2) can be truncated at $n=2$ and $F_{2}^{(2)}\left(Q^{2}\right)$ comprises contributions from all poles with $n \geq 2$.

The BFKL equation allows one to determine the intercepts and structure functions $F_{2}^{(n)}\left(Q^{2}\right)$. The only adjustable parameters are "the pole residues" $A_{0}, A_{1}, A_{2}$ in (2) which are fixed, in fact, by the boundary condition at $x=x_{0}$. With the proper account of the valence [9] and non-perturbative [8] corrections to (2) we arrive at the three-pole-approximation which appears to be very successful when confronted with the data [10]. in a wide kinematical range (Figure 3). The effective pomeron intercept

$$
\Delta_{e f f}=-\frac{\partial \log F_{2 p}\left(x, Q^{2}\right)}{\partial \log x}
$$

gives an idea of the role of the subleading singularities. The intercept $\Delta_{\text {eff }}$ calculated with the experimental kinematic constraints is much smaller than $\Delta_{0}=0.4$ which is expected to dominate asymptotically. The agreement of our numerical estimates with the $H 1$ determination (Figure 4 ) is quite satisfactory.

Acknowledgments I would like to thank José Repond for the kind hospitality in Chicago and careful reading the manuscript.

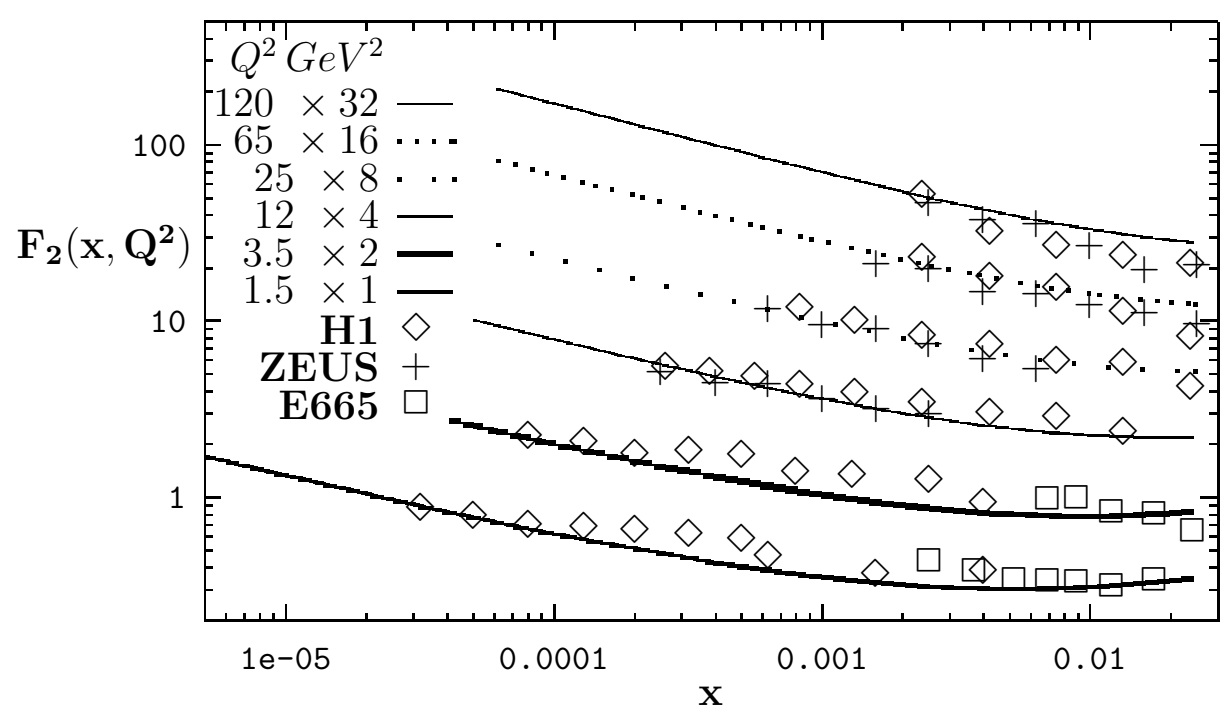

FIGURE 3. Three-Pole Approximation vs. H1, ZEUS and E665 data. 


\section{REFERENCES}

1. Gribov V.N., and Lipatov L.N., Sov. J. Nucl. Phys. 15, 438 (1972); Dokshitzer Yu.L., Sov. Phys. JETP 46, 641 (1977); Altarelli G., and Parisi G., Nucl. Phys. B126, 297 (1977).

2. Fadin V.S., Kuraev E.A.,and Lipatov L.N., Sov. Phys. JETP 44, 443 (1976); ibid 45, 199 (1977).

3. Lipatov L.N., Sov. Phys. JETP 63, 904 (1986)

4. Nikolaev N.N., Zakharov B.G., and Zoller V.R., JETP Letters 59, 8 (1994).

5. Nikolaev N.N., Zakharov B.G., Phys. Letters B327, 157 (1994).

6. Karl G., and Novikov V. Phys. Rev D51, 5069 (1995).

7. Nikolaev N.N., Zakharov B.G., and Zoller V.R., JETP Letters 60, 678 (1994).

8. Nikolaev N.N., and Zakharov B.G., Phys. Letters B333, 250 (1994).

9. Glück M., Reya E., and Vogt A, Z. Phys. C67, 433 (1995).

10. H1 Collab., Nucl. Phys. B439, 471 (1995); ZEUS Collab., Z. Phys. C69, 607 (1996); E665 Collab., Phys. Rev. D54, 3006 (1996).

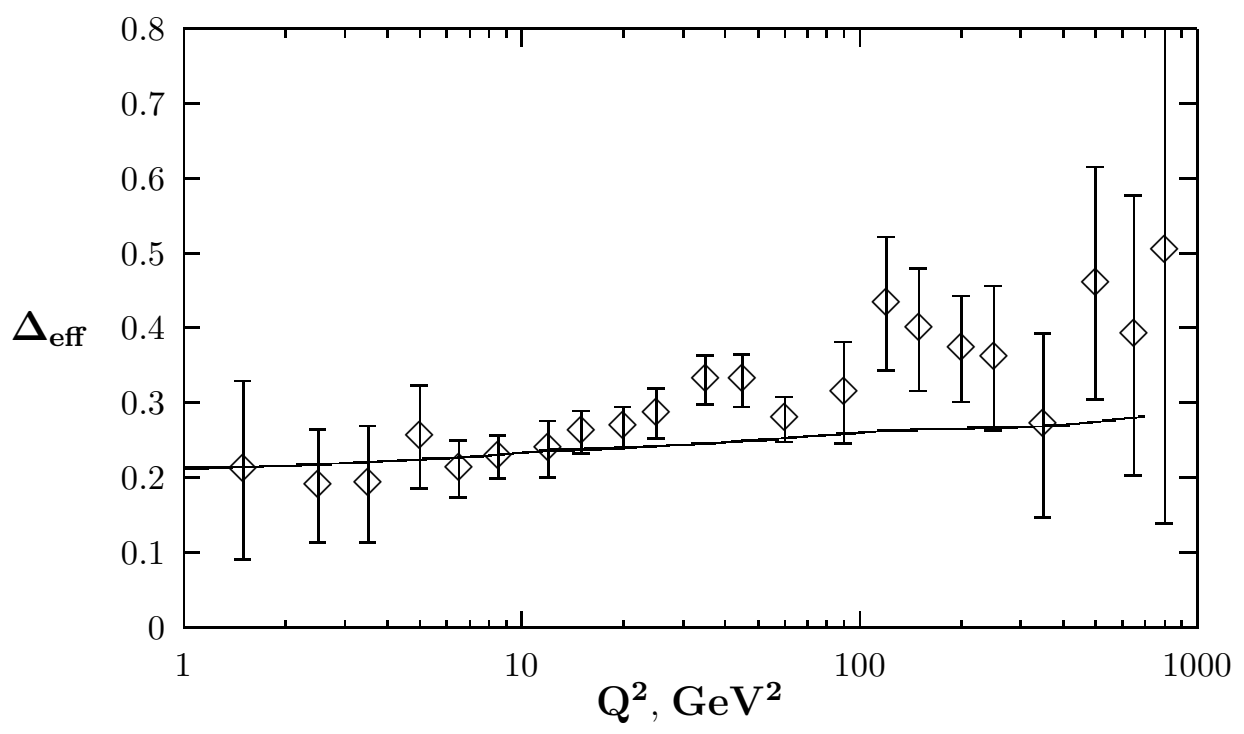

FIGURE 4. Effective intercept vs. H1 data. 